\title{
Increasing the Dimensionality of In-situ Electron Microscopy Data Sets by On-the- fly and Analytical Electron Tomography
}

\author{
Ruoqian Lin ${ }^{1}$, Lili Han ${ }^{1}$ and Huolin L. Xin ${ }^{1}$ \\ 1. Center for Functional Nanomaterials, Brookhaven National Laboratory, Upton, NY
}

The spatial, compositional, bonding, and time-domain complexity of materials transformation under the solid/gas reaction [1-3], particularly with heterogeneous nucleation involved, highlights the need to increase the dimensionality of electron microscopy data sets beyond conventional projection imaging and the acquisition of image series, i.e. $x$ - $y$-t. Because projection images can be misleading or inconclusive for inhomogeneous systems, it is much desired to include all three spatial coordinates, $x, y$, and $z$, into the data set without losing the time and energy resolution. However, a key stumbling block that had held back progress in achieving this is the rather slow process in acquiring electron tomography data for the retrieval of depth information. In this abstract, we will report the realization of highthroughput electron tomography, on-the-fly reconstruction, and the first attempt to achieve 5-D electron microscopy data sets under reaction conditions.

The most time consuming step in traditional tomography data acquisition is the tracking and settling of the objects due to the mechanical shift/drift at each tilt increment. However, with high-frame-rate live imaging available now in both TEM and STEM, projection images with high resolution can be recovered even when the objects is moving in the field of view. Using non-rigid registration and iterative corrections between the 3-D coordinates and the radon space, the live recorded data can be fully utilized and is sufficient for high-quality reconstructions. This makes every electron that goes through the sample be used for 3-D reconstruction, which does not only shorten the tomography measurement time from 2-3 hours down to 10 minutes, also does it improve the dose efficiency of such experiments. We have successfully demonstrated that by following the algorithm shown in Figure 1, we can enable on-the-fly reconstructions of nanoobjects. For instance, it takes less than 20 seconds for a workstation to automatically reconstruct the faceted nanoparticles in Fig. 1 starting from the original unprocessed liveview tilt series.

Fig. 2 demonstrates the capability to extend to five-dimensional data sets to visualize the oxidation of a Co-Fe catalyst with unprecedented 3-D and chemical details using STEM-EELS tomography. Compared with STEM-EDX, the advantage of STEM-EELS is its capacity to extract spatially resolved bonding information. By applying statistical classification to the Fe L2,3 near edge fine structures, the metallic and oxidized iron component can be readily reconstructed in 3-D (red and green in Fig. 2). In addition, one aspect that EELS traditionally falls short of is that EELS has difficulty working with thick samples due to multiple inelastic scattering. We used a quantitative dual-EELS method developed in refs. [4] to correct for this artifact in the tilt series. The fulfilment of the projection requirement not only improves the reconstruction resolution but also allows tomograms to be rendered quantitatively. [5]

References:

[1] R. Sharma and K. Weiss, Microscopy Research and Technique 42 (4), 270 (1998).

[2] HL Xin et al, Microscopy and Microanalysis 19 (06), 1558 (2013).

[3] HL Xin et al, Nano Letters 14 (6), 3203 (2014).

[4] HL Xin et al, Utramicroscopy, 19, 38 (2014) and HL Xin et al, Phys. Rev. B., 90, 214305 (2014) 
[5] Research carried out at the CFN/BNL, which is supported by the U.S. Department of Energy, Office of Basic Energy Sciences, under Contract No. DE-SC0012704.

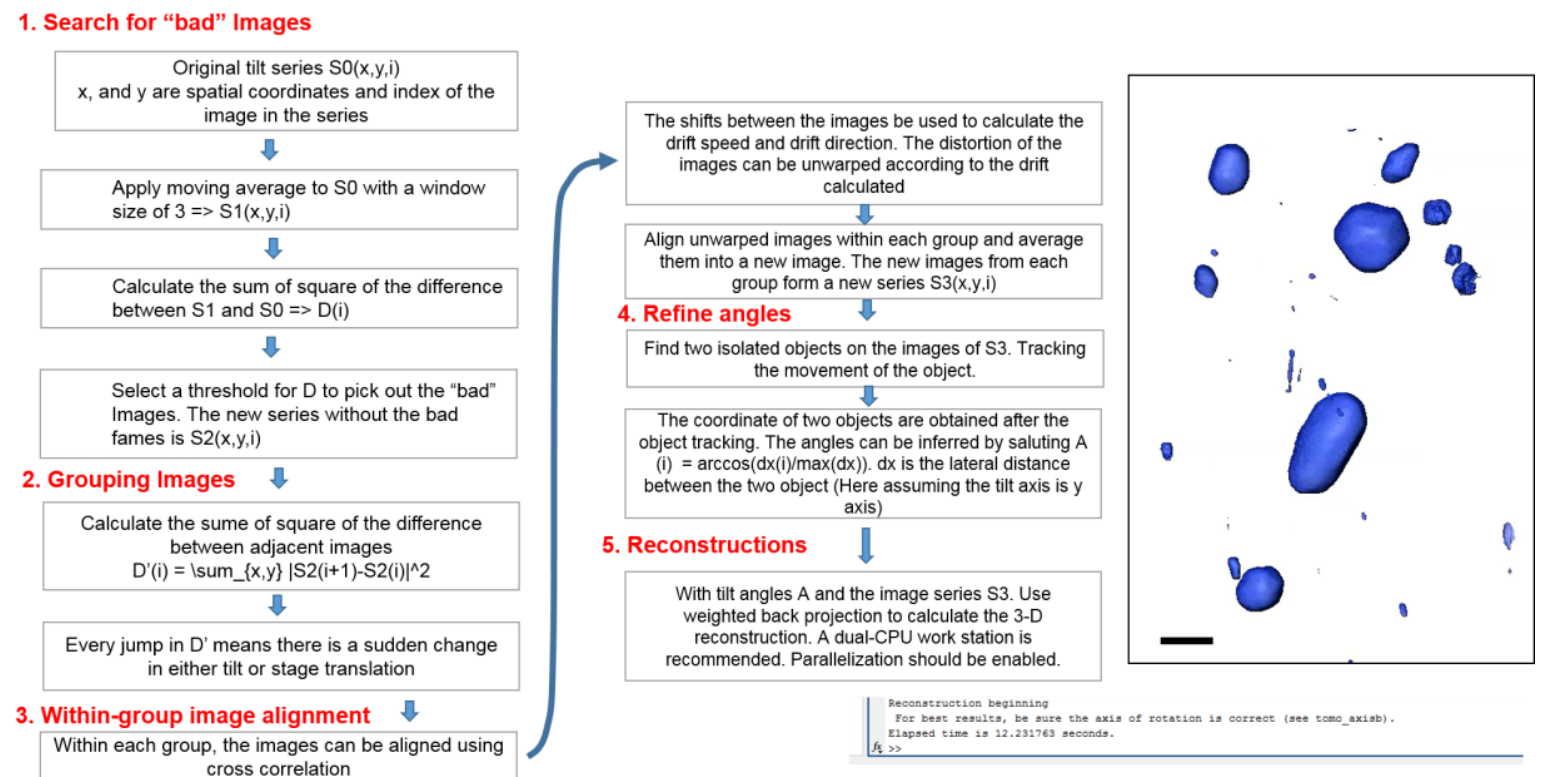

Figure 1. The algorithm for the one-the-fly reconstruction and the three-dimensional rendering of the particle reconstructed from a high-throughput data set that took less than 10 minutes to acquire. (Scale bar: $100 \mathrm{~nm})$.

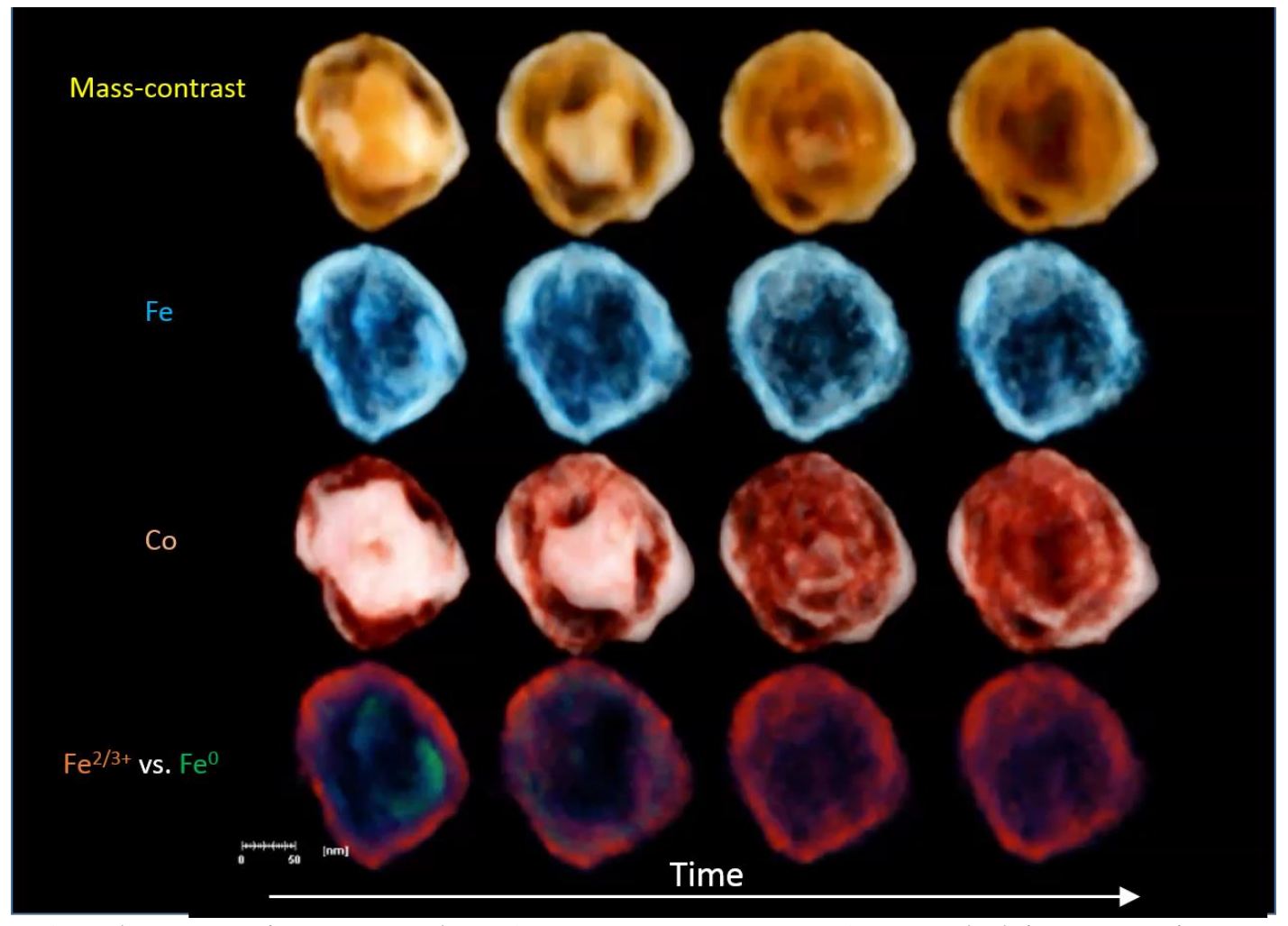

Figure 2. 5D electron microscopy data ( $x, y, z$, vs. $E_{\text {loss }}$ vs. $t$ ) recorded in an environmental TEM tracking the oxidation process of a Co-Fe catalyst with three-dimensional and chemical details. (Scale bar: $50 \mathrm{~nm}$ ) 